\title{
Effects of confinement on the vibrational spectra of liquid water adsorbed in carbon nanotubes
}

\author{
J. Martí* \\ Departament de Física i Enginyeria Nuclear, Universitat Politècnica de Catalunya, B5 Campus Nord, 08034 Barcelona, Catalonia, Spain \\ M. C. Gordillo \\ Departamento de Ciencias Ambientales, Facultad de Ciencias Experimentales, \\ Universidad Pablo de Olavide. Carretera de Utrera Km 1, 41013 Sevilla, Spain \\ and Departament de Física i Enginyeria Nuclear, Universitat Politècnica de Catalunya, B5 Campus Nord, \\ 08034 Barcelona, Catalonia, Spain \\ (Received 25 January 2001; published 5 April 2001)
}

\begin{abstract}
We report the results of a calculation of the vibrational and rotational spectra of water confined in carbon nanotubes. Water has been modeled with a flexible version of the simple point charge model of Berendsen et al., while the carbon nanotubes are taken to be soft potential walls interacting with water through a LennardJones-type potential. The comparison of the computed absorption line shapes with the corresponding ones of liquid bulk water shows significant shifts in the positions of the spectral bands directly related to the tube radii.
\end{abstract}

DOI: 10.1103/PhysRevB.63.165430

PACS number(s): 31.15.Qg, 36.20.Ng, 68.35.Ja, 73.20.Mf

\section{INTRODUCTION}

Carbon nanotubes have recently received a lot of attention because of their interesting properties in a variety of fields. ${ }^{1-5}$ In particular, it has been observed that single wall carbon nanotubes (SWCN) are quasi-one-dimensional structures with unique absorption capabilities. On the other hand, even though water has been extensively studied, some of its properties still need to be completely understood. ${ }^{6}$ A significant number of them are related to its behavior under confinement. This is a very important issue, since water is totally or partially constrained in many scientifically relevant situations, such as in biological cells, in the surface of lipid membranes and proteins, or in most of geological systems. Very recently, theoretical and experimental work has been done in water confined in silica pores ${ }^{7-9}$ but, to our knowledge, there are no experimental data for narrower pores like those we can observe in some carbon nanotubes. The closest information would be data obtained from far-infrared rotationalvibrational tunneling spectroscopy in water clusters, ${ }^{10}$ since the width of the tubes could be comparable to the total width of the cluster. Structure and the hydrogen-bond network of water adsorbed in SWCN has been recently studied by means of molecular dynamics (MD) simulations, ${ }^{11}$ so in this paper we will concern ourselves basically with dynamical properties.

\section{COMPUTATIONAL DETAILS}

We consider the role played by confinement on the spectra of liquid water adsorbed in carbon nanotubes. To observe the influence of the radius of the tube on the studied properties, we performed a series of MD simulations of liquid water in different tubes, all of them at $298 \mathrm{~K}$ and for a water density of $1 \mathrm{~g} / \mathrm{cm}^{3}$. Such a density is the value that we wish to simulate, although we have observed in several simulations at fixed temperature and variable density (about 20\% up or down $1 \mathrm{~g} / \mathrm{cm}^{3}$ ) that all properties remain qualitatively unchanged. Water-water interactions were modeled with a flexible version ${ }^{12}$ of the simple point charge (SPC) potential. ${ }^{13}$ This potential includes intramolecular interactions composed by harmonic and Morse-type terms and is able to reproduce the main trends of the experimental frequency bands associated with the infrared spectrum of bulk water. ${ }^{14}$ Within this approximation, we can exactly reproduce the positions of the band maxima for each of the spectral regions, since the fundamental frequency of a quantum oscillator is the same as that of a classical one. However, we can get neither the heights nor the widths of the real spectrum bands.

We considered four types of SWCN, $(6,6),(8,8),(10,10)$, and $(12,12)$ in the standard nomenclature, ${ }^{15}$ which correspond to cylindrical tubes of radii 4.10, 5.45, 6.80, and $8.15 \AA$, respectively (see Table I). These are not the narrowest tubes experimentally obtained: one can even get $(5,5)$ and narrower tubes. ${ }^{16,17}$ Water-tube interactions were described by a soft potential wall of the Lennard-Jones type. ${ }^{18}$ This is an averaged potential; we do not consider each carbon in the tube, but the average interaction of all of them with the water molecules. In particular, there is no dependence of the interaction on the $z$ position of the water, since the potential is averaged to depend only on the distance to the center of the tube. However, the particular form of this potential is not important but the tube radius that defines the confinement. Thus, we obtained very similar results when we used a confining rigid cylinder. Carbon-oxygen and carbon-hydrogen $\sigma$ and $\epsilon$ parameters have been obtained from Lorentz-Berthelot

TABLE I. Simulated systems. All simulations have been performed at $298 \mathrm{~K}$ and $1.00 \mathrm{~g} / \mathrm{cm}^{3}$.

\begin{tabular}{cccc}
\hline \hline Simulation & Tube radius $(\AA)$ & Tube class & $\begin{array}{c}\text { Number of } \\
\text { water molecules }\end{array}$ \\
\hline 1 & 4.10 & $(6,6)$ & 14 \\
2 & 5.45 & $(8,8)$ & 56 \\
3 & 6.80 & $(10,10)$ & 126 \\
4 & 8.15 & $(12,12)$ & 224 \\
\hline \hline
\end{tabular}


mixing rules. ${ }^{19}$ The values we employed are: $\sigma_{\mathrm{OC}}=3.28 \AA$, $\sigma_{\mathrm{HC}}=2.81 \AA, \epsilon_{\mathrm{OC}}=46.79 \mathrm{~K}, \epsilon_{\mathrm{HC}}=15.52 \mathrm{~K}$. The internal radii of each SWCN obtained with this model are 1.31, 2.65, 4.00 , and $5.33 \AA$. The length of all carbon nanotubes was $74.5 \AA$. All these geometrical characteristics are suitable to model liquid water inside SWCN since we have fixed the liquid water density as one of our input values and obtained the number of water molecules for each tube as an output to fix our MD initial configurations. It should be pointed out that experimental densities (when available) could be slightly different from that one used in this work. On the other hand, the configurational energies of adsorbed water fluctuate around $-11 \mathrm{kcal} / \mathrm{mol}$ for all tube classes, whereas the value for bulk water at ambient conditions is about $-10 \mathrm{kcal} / \mathrm{mol}$. This is an indication of the stability of the MD simulations we report.

Standard periodic boundary conditions and the Ewald sum rule were used to compute long-ranged Coulombic forces. Our MD integration algorithm was a leapfrog Verlet form with coupling to a Berendsen thermostat. ${ }^{20} \mathrm{We}$ applied a time step of $0.5 \mathrm{fs}$. In addition, the translational and internal degrees of freedom were independently equilibrated. ${ }^{21}$ We performed MD runs of length $0.5 \mathrm{~ns}$ to collect statistics, after equilibration periods of $0.1 \mathrm{~ns}$.

\section{SPECTRAL DENSITIES}

The experimental infrared spectrum is usually measured by means of the absorption coefficient $\alpha(\omega)$ or of the imaginary part of the dielectric constant $\varepsilon^{\prime \prime}(\omega) .^{22}$ These are quantum properties that can be approximately computed in a classical framework by making use of some hypotheses. The significant object that has to be calculated is the absorption line shape $I(\omega)$, related to $\alpha(\omega)$ and $\varepsilon^{\prime \prime}(\omega)$ in the following way: ${ }^{14}$

$$
\begin{gathered}
\alpha(\omega)=\frac{4 \pi^{2} \omega}{3 \hbar c n V}[1-\exp (-\beta \hbar \omega)] I(\omega), \\
\varepsilon^{\prime \prime}(\omega)=\frac{n c \alpha(\omega)}{\omega},
\end{gathered}
$$

where $n$ is the refractive index, $V$ is the volume of the system, and $I(\omega)$ can be calculated through the normalized hydrogen velocity autocorrelation function $C_{\mathrm{H}}(t)$ given by

$$
C_{\mathrm{H}}(t)=\frac{\left\langle\vec{v}_{\mathrm{H}}(t) \cdot \vec{v}_{\mathrm{H}}(0)\right\rangle}{\left\langle\vec{v}_{\mathrm{H}}^{2}(0)\right\rangle} .
$$

The final form ${ }^{14}$ of $I(\omega)$ is

$$
I(\omega)=2 \pi^{-1} N q^{2} \omega^{-2} \int_{0}^{\infty} d t C_{\mathrm{H}}(t) \cos \omega t
$$

where $N$ is the number of molecules of the system and $q$ is the hydrogen charge. Since the relevant quantity is $I(\omega)$, we will compute it in arbitrary units for each spectral region and

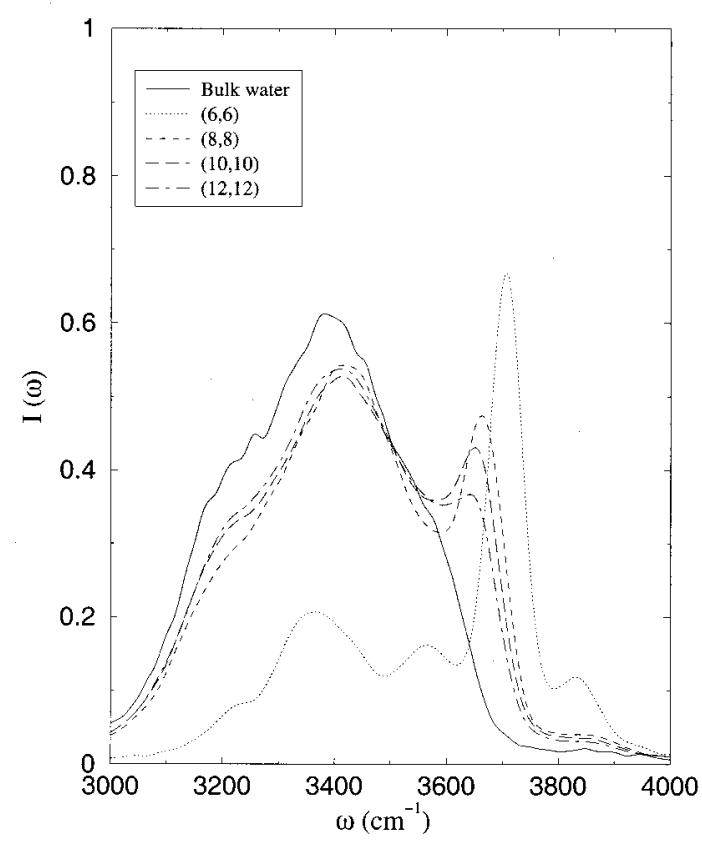

FIG. 1. Comparison of the hydrogen absorption line shape (arbitrary units) for bulk water and water confined in carbon nanotubes between 3000 and $4000 \mathrm{~cm}^{-1}$.

compare the results with the bulk data. To calculate the Fourier transform, a Hanning window together with a Bode rule were employed. ${ }^{23,24}$

\section{DISCUSSION}

We displayed in Fig. 1 the hydrogen absorption line shape (arbitrary units) in the stretching region for bulk water compared with the same function for confined water in carbon nanotubes. To our knowledge, no spectroscopic measures of infrared nor Raman spectra of liquid water in carbon nanotubes are available to compare. The most important feature we observe is the presence of a frequency band between 3640 and $3690 \mathrm{~cm}^{-1}$ (absent in the bulk spectrum), which we call "vibration." The position of this new vibrational band shifts to lower values (referred to the bulk stretching mode) when the tube radius increases. This new feature cannot be straightforwardly interpreted as an overtone of the ordinary vibrational bands (bending, stretching). ${ }^{25}$ Further, we observed the same feature in simulations of water inside rigid cylinders as well as in deuterated and in tritium water. ${ }^{26}$

In our opinion, the presence of the vibration band is purely an effect of confinement and can be explained by the splitting of the symmetric and antisymmetric components of the $\mathrm{O}-\mathrm{H}$ stretching vibration together with the displacement of that stretching frequency to gas phase values, which are, in general, greater. In the absence of confinement, only the latter circumstance is produced and we have a unique peak, not two, at larger wave numbers. ${ }^{27,28}$ This explanation for our results can be backed up with the following data. First, the experimental values of the gas phase symmetric and antisymmetric stretching frequencies are about 3656 and 
TABLE II. Frequency maxima and absolute values of frequency shifts for rotation, stretching and vibration referred to bulk water, for water confined in carbon nanotubes. Bulk water data are taken from Ref. 27. The estimated statistical uncertainty is around $10 \mathrm{~cm}^{-1}$ for all values.

\begin{tabular}{cccccc}
\hline \hline Simulation & $\omega_{\mathrm{R}}\left(\mathrm{cm}^{-1}\right)$ & $\Delta \omega_{\mathrm{R}}\left(\mathrm{cm}^{-1}\right)$ & $\omega_{\mathrm{S}}\left(\mathrm{cm}^{-1}\right)$ & $\omega_{\mathrm{V}}\left(\mathrm{cm}^{-1}\right)$ & $\Delta \omega_{\mathrm{V}}\left(\mathrm{cm}^{-1}\right)$ \\
\hline 1 & 400 & 91 & 3363 & 3707 & 325 \\
2 & 420 & 71 & 3420 & 3662 & 280 \\
3 & 448 & 43 & 3412 & 3657 & 275 \\
4 & 472 & 19 & 3411 & 3641 & 259 \\
Bulk & 491 & 0 & 3382 & - & 0 \\
\hline \hline
\end{tabular}

$3755 \mathrm{~cm}^{-1}$, respectively, ${ }^{29}$ while in MD simulations of liquid water these values are about 3400 and $3600 \mathrm{~cm}^{-1}$, respectively. Second, the hydrogen-bond network of water confined in SWCN is markedly weaker than that in bulk systems, ${ }^{11}$ so the number of non-H-bonded hydrogens in the former increases and a shift to gas phase values is produced. We can consider that our actual "stretching" frequency (Fig. 1 and Table II) would be the symmetric stretching component indeed, whereas what we call the "vibration", frequency would really be the antisymmetric component of the bulk stretching frequency. The rise of the antisymmetric stretch would be due to the $20 \%$ of water molecules forming only one or even zero $\mathrm{H}$ bonds. ${ }^{11}$

We also reported the absorption line shape in the domain between 300 and $1000 \mathrm{~cm}^{-1}$, which corresponds to rotational motions of the water molecule (Fig. 2). Again a shift to lower frequencies than those in the bulk is observed. We also observe the rise of an additional high-frequency peak around $650 \mathrm{~cm}^{-1}$ in the case of the $(6,6)$ tube, which could be attributed to an optical mode transmitted along the whole hydrogen-bond network, in a similar fashion to the optical

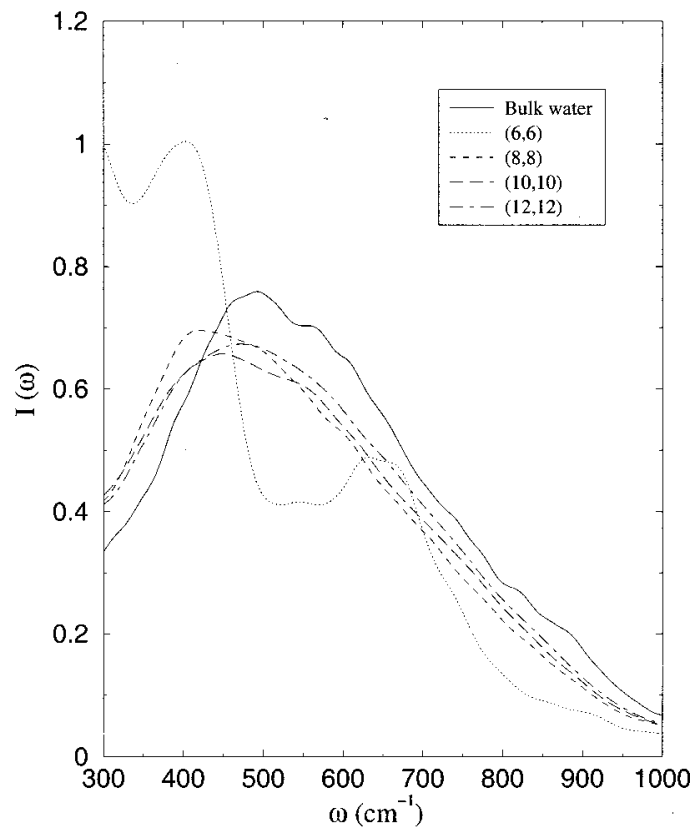

FIG. 2. Same as Fig. 1 for frequencies between 300 and $1000 \mathrm{~cm}^{-1}$. mode found in bulk water around $685 \mathrm{~cm}^{-1} \cdot{ }^{14,30}$ It is remarkable to note that in the middle-frequency range, which corresponds to the bending vibrations of water (Fig. 3), the shifts are basically of the same magnitude and they are independent of the tube radius.

To our surprise, we observed that there was a direct relationship between the radii of the tubes and some of the shifts mentioned above. For instance, we found that

$$
\Delta \omega_{\mathrm{R}}=a_{\mathrm{R}}+b_{\mathrm{R}} r
$$

with $a_{\mathrm{R}}=167.4 \pm 3.0 \mathrm{~cm}^{-1}$ and $b_{\mathrm{R}}=-18.1 \pm 0.5 \mathrm{~cm}^{-1} / \AA$ for the rotational frequency band shift and

$$
\Delta \omega_{\mathrm{V}}=a_{\mathrm{V}}+b_{\mathrm{V}} / r
$$

with $a_{\mathrm{V}}=194 \pm 2 \mathrm{~cm}^{-1}$ and $b_{\mathrm{V}}=524 \pm 7 \AA \mathrm{cm}^{-1}$ for the vibrational frequency band shift. In both cases, the $\chi^{2}$ parameter per degree of freedom is less than 1. On the other hand, the changes in the position of other bands are not so easily related to the tube radii. This is the case of the stretching frequencies (centered around $3380 \mathrm{~cm}^{-1}$ in bulk water) and

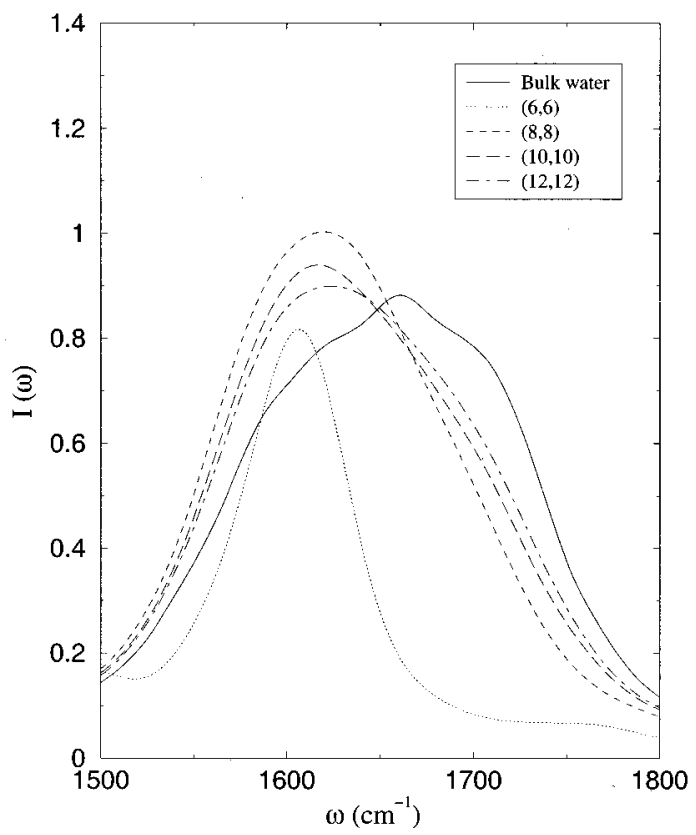

FIG. 3. Same as Figs. 1 and 2 for frequencies between 1500 and $1800 \mathrm{~cm}^{-1}$. 


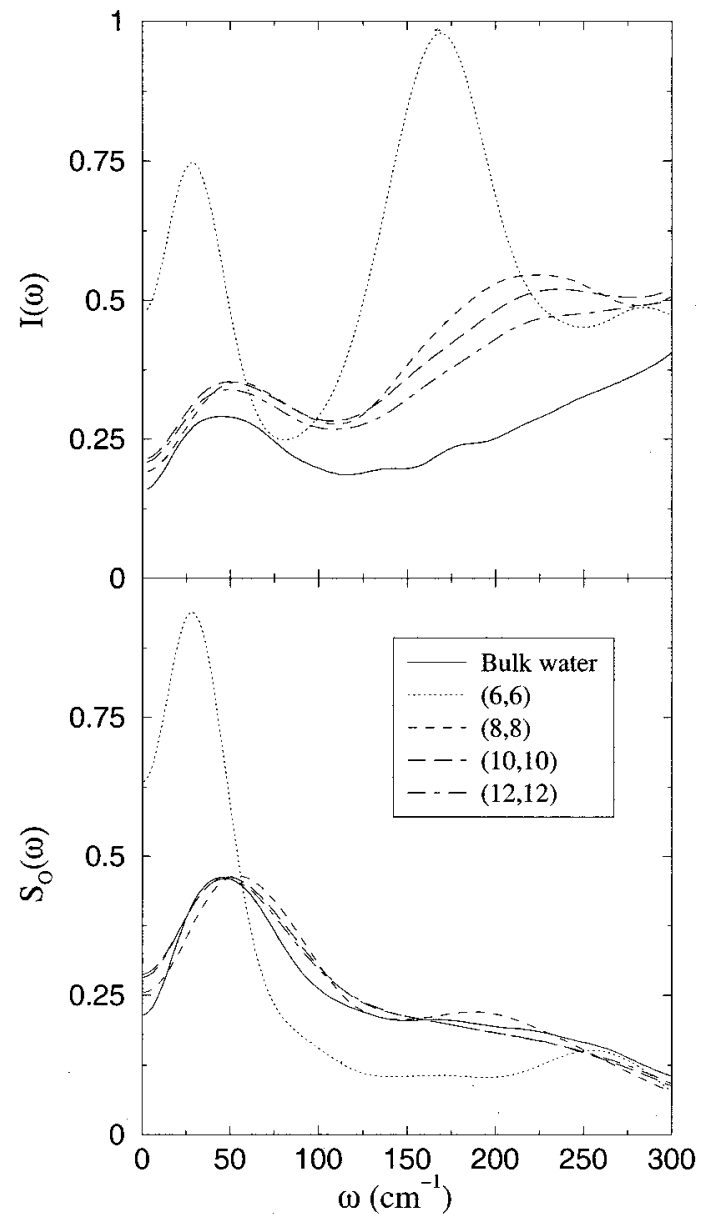

FIG. 4. Same as Figs. 1, 2, and 3 for frequencies between 0 and $300 \mathrm{~cm}^{-1}$ (top). Comparison of the spectral densities of the oxygen velocity autocorrelation functions for bulk water and water confined in carbon nanotubes (bottom).

the bending ones (centered around $1660 \mathrm{~cm}^{-1}$ in bulk water), which are slightly displaced to lower values with respect to the bulk (see Table II). Nevertheless, both Eqs. (4) and (5) would allow us, in principle, to obtain the radius of a nanotube by measuring the spectrum of confined water inside it.

The low-frequency domain of $0-300 \mathrm{~cm}^{-1}$ was analyzed from two complementary calculations shown in Fig. 4. We employed the low-frequency range of the absorption line shape and the spectral density $S_{\mathrm{O}}(\omega)$, computed as the Fourier transform of the oxygen velocity autocorrelation function. This spectral density cannot be related to any observable quantity but it is the basic quantity employed in most MD simulations to study hindered translations and intermolecular vibrations. ${ }^{31}$ These kind of motions have been the subject of debate for a long time. Walrafen ${ }^{32}$ argued that the two relevant features appearing in the low-frequency spectrum of liquid bulk water are concerned with local effects associated with the hydrogen-bond network: the stretching of hydrogen bonds (high-frequency band around $200 \mathrm{~cm}^{-1}$ ) and the bending of hydrogen-bonded O-O-O units (low- frequency band around $50 \mathrm{~cm}^{-1}$ ). The scientific community has widely agreed about the $200 \mathrm{~cm}^{-1}$ band, whereas there is no general agreement about the $50 \mathrm{~cm}^{-1}$ peak. ${ }^{27,33,34}$ The alternative to the standard interpretation is to consider the low-frequency band as the result of the cage effect of the neighboring water molecules. ${ }^{27}$

We found significant differences between the position of both frequency bands in bulk water and in confined liquid in the narrow $(6,6)$ and $(8,8)$ tubes. In the case of the broader $(10,10)$ and $(12,12)$ tubes, differences with bulk are very small. In the $(6,6)$ tube, we observe that the low-frequency peak was displaced to $30 \mathrm{~cm}^{-1}$. Thus, we should note that $\mathrm{O}-\mathrm{O}-\mathrm{O}$ units are difficult to produce in this linear environment. In addition, the high-frequency band appears very intense in the absorption line shape of the $(6,6)$ tube, around $170 \mathrm{~cm}^{-1}$. The spectral density $S_{\mathrm{O}}(\omega)$ reveals the same behavior of the low-frequency band but the $170 \mathrm{~cm}^{-1}$ band virtually disappears. Instead, it shows up as a second highfrequency band centered around $260 \mathrm{~cm}^{-1}$, which appears to be much less intense in the absorption line shape. These two peaks could be attributed to the collective vibrations of the basically linear hydrogen-bond network. ${ }^{11}$ When the nanotube radius increases, this network becomes more and more three-dimensional, as in bulk water, and the spectra transforms accordingly. However, we can still see some effects in the $(8,8)$ tube, in which there is a redshift of the highfrequency peak directly related to the tube radius. On the other hand, in this tube, the low-frequency band does not show significant differences with bulk water.

\section{CONCLUSIONS}

We have performed a series of molecular dynamics simulations of liquid water adsorbed in carbon nanotubes at ambient conditions. Our results allow us to observe a new highfrequency vibration peak around $3650 \mathrm{~cm}^{-1}$ in the midinfrared spectrum of liquid water confined in carbon nanotubes. That frequency can be attributed to the splitting of the symmetric and antisymmetric components of the stretching vibration of liquid water. Second, we have observed frequency shifts of two spectral bands that can be directly related to the tube radii. These spectroscopic displacements could be the basis of an experimental procedure for characterizing carbon nanotubes. We hope that the experimental measure of the Raman and the infrared spectrum of confined water in carbon nanotubes could confirm these findings.

\section{ACKNOWLEDGMENTS}

This work has been partially supported by the Direcció General de Recerca de la Generalitat de Catalunya, Project No. 1999SGR-00146, the Ministerio de Educación y Cultura of Spain, Grants No. PB96-0170-C03-02 and No. PB980922, and Project No. PR99-05 of the Universitat Politècnica de Catalunya. M.C.G. also thanks the Spanish Ministry of Education and Culture for additional support. 
*Corresponding author. Electronic address: jordi.marti@upc.es

${ }^{1}$ R. Saito, G. Dresselhaus, and M.S. Dresselhaus, Physical Properties of Carbon Nanotubes (Imperial College Press, London, 1998).

${ }^{2}$ M.S. Dresselhaus, G. Dresselhaus, P.C. Eklund, and R. Saito, Phys. World 11, 33 (1998).

${ }^{3}$ U.D. Venkateswaran, A.M. Rao, E. Richter, M. Menon, A. Rinzler, R.E. Smalley, and P.C. Eklund, Phys. Rev. B 59, 10928 (1999).

${ }^{4}$ D. Kahn and J.P. Lu, Phys. Rev. B 60, 6535 (1999).

${ }^{5}$ Z. Yao, H.W.Ch. Postma, L. Balents, and C. Dekker, Nature (London) 402, 273 (1999).

${ }^{6} \mathrm{~F}$. Franks, in Water: A Comprehensive Treatise, edited by F. Franks (Plenum Press, New York, 1972).

${ }^{7}$ F. Bruni, M.A. Ricci, and A.K. Soper, J. Chem. Phys. 109, 1478 (1998)

${ }^{8}$ A.K. Soper, F. Bruni, and M.A. Ricci, J. Chem. Phys. 109, 1486 (1998).

${ }^{9}$ C. Hartnig, W. Witschel, E. Spohr, P. Gallo, M.A. Ricci, and M. Rovere, J. Mol. Liq. 85, 127 (2000).

${ }^{10}$ K. Liu, J.D. Cruzan, and R.J. Saykally, Science 271, 929 (1996).

${ }^{11}$ M.C. Gordillo and J. Martí, Chem. Phys. Lett. 329, 341 (2000).

${ }^{12}$ J. Martí, J.A. Padró, and E. Guàrdia, J. Mol. Liq. 62, 17 (1994).

${ }^{13}$ H.J.C. Berendsen, J.P.M. Postma, W.F. van Gunsteren, and J. Hermans, in Intermolecular Forces, edited by B. Pullman (Reidel, Dordretct, Holland, 1981).

${ }^{14}$ J. Martí, E. Guàrdia, and J.A. Padró, J. Chem. Phys. 101, 10883 (1994).

${ }^{15}$ N. Hamada, S. Isawada, and A. Oshiyama, Phys. Rev. Lett. 68, 1579 (1992).
${ }^{16}$ S. Iijima and T. Ichiihashi, Nature (London) 363, 603 (1993).

${ }^{17}$ L.F. Sun, S.S. Xie, W. Liu, W.Y. Zhou, Z.Q. Liu, D.S. Tang, G. Wang, and L.X. Qian, Nature (London) 403, 384 (2000).

${ }^{18}$ G. Stan and M.W. Cole, Surf. Sci. 395, 280 (1997).

${ }^{19}$ K.G. Ayappa and V. Shankar, Electrochem. Soc. Proc. 95-10, 1489 (1995)

${ }^{20}$ H.J.C. Berendsen, J.P.M. Postma, W.F. van Gunsteren, A. DiNola, and J.R. Haak, J. Phys. Chem. 81, 3684 (1984).

${ }^{21}$ A. Wallqvist and O. Telemann, Mol. Phys. 74, 515 (1991).

${ }^{22}$ D.A. McQuarrie, Statistical Mechanics (Harper and Row, New York, 1976).

${ }^{23}$ J. Martí, Ph.D. thesis, University of Barcelona, 1995.

${ }^{24}$ M.P. Allen and D.J. Tildesley, Computer Simulation of Liquids (Clarendon, Oxford, 1987).

${ }^{25}$ J. Martí, J.A. Padró, and E. Guàrdia, Mol. Simul. 11, 336 (1993).

${ }^{26}$ J. Martí and M.C. Gordillo J. Chem. Phys. (to be published).

${ }^{27}$ J. Martí, J.A. Padró, and E. Guàrdia, J. Chem. Phys. 105, 639 (1996).

${ }^{28}$ J. Martí, J. Chem. Phys. 110, 6876 (1999).

${ }^{29}$ S.H. Chen, K. Toukan, C.K. Loong, D.L. Price, and J. Teixeira, Phys. Rev. Lett. 53, 1360 (1984).

${ }^{30}$ H. Resat, F.O. Raineri, and H.L. Friedman, J. Chem. Phys. 97, 2618 (1992).

${ }^{31}$ J. Martí, Phys. Rev. E 61, 449 (2000).

${ }^{32}$ G.E. Walrafen, in Water: A Comprehensive Treatise, edited by F. Franks (Plenum Press, New York, 1972).

${ }^{33}$ G.E. Walrafen, Y.C. Chu, and G.J. Piermarini, J. Phys. Chem. 100, 10363 (1996).

${ }^{34}$ T. Nakayama, Phys. Rev. Lett. 80, 1244 (1998). 